\title{
The Liver and Kidney in Critically III Patients
}

\author{
Andrew J. Slack Julia Wendon \\ The Institute of Liver Studies, KCH, King's College London, King's College Hospital, London, UK
}

\section{Key Words}

Hepatorenal syndrome $\cdot$ Acute kidney injury $\cdot$ Renal blood

flow $\cdot$ Cirrhotic cardiomyopathy

\begin{abstract}
Both liver and kidney dysfunction are associated with adverse outcomes in critical illness. Advanced liver disease can be complicated by the hepatorenal syndrome (HRS) with liver transplantation offering the best long-term outcome. However, until recently, HRS was associated with such a poor prognosis that this group of patients rarely survived long enough for transplantation to be considered. The use of vasopressin analogues and albumin infusions has improved the management of HRS and outcomes in terms of renal recovery and survival.

Copyright $\odot 2009$ S. Karger AG, Basel
\end{abstract}

\section{Introduction}

Preservation of organ function is the keystone in the survival of critically ill patients on intensive care. It has been well reported that coexisting and indeed independent liver and renal dysfunction impact significantly on hospital and intensive care mortality [1]. For over 100 years the association between liver and kidney disease has been well documented. In 1877, the clinical phenomenon of oliguria, ascites and normal kidney histology was noted by Frerichs, a German pathologist. He went on to also describe the pathological changes seen in cirrhosis of the liver $[2,3]$. The term hepatorenal syndrome (HRS) was first used in 1939 to describe kidney dysfunction following biliary surgery and liver trauma. HRS is a unique form of renal dysfunction that complicates advanced liver disease, acute hepatic failure or portal hypertension. The key pathological features are the development of cirrhotic cardiomyopathy and vasoactive mediator-induced circulatory dysfunction or 'vascular failure'. HRS is a diagnosis of exclusion with other common causes of acute renal failure (ARF), including pre-renal, renal and postrenal causes also seen frequently in advanced liver disease. We will explore these and focus on HRS, which despite its functional nature, continues to be associated with a poor prognosis.

\section{Epidemiology}

UK-based population studies have estimated the annual incidence of ARF from 486 to 620 per million population, accounting for about $1 \%$ of hospital admissions and complicating $>7 \%$ of hospital inpatient admissions $[4,5]$. ARF is a common finding on the critical care unit with as many as $35 \%$ of patients admitted to ICU requiring renal replacement therapy (RRT) and, when present as part of multiple organ failure, mortality is $>90 \%$ if four organs or more fail [6].

The commonest forms of ARF in cirrhotic patients with ascites are pre-renal failure and acute tubular necro-

\section{KARGER \\ Fax +4161306 1234 \\ E-Mail karger@karger.ch}

www.karger.com
(C) 2009 S. Karger AG, Basel

0253-5068/09/0282-0124\$26.00/0

Accessible online at:

www.karger.com/bpu
Andrew J. Slack

The Institute of Liver Studies, KCH, King's College London

Denmark Hill, London SE5 9RS (UK)

E-Mail doctorslack@hotmail.com 
sis (ATN) with prevalence rates of 42 and $38 \%$, respectively. Post-renal failure occurs at a rate of $0.3 \%$. HRS occurs in about $4 \%$ of patients admitted with decompensated cirrhosis, the cumulative probability being $18 \%$ at 1 year, increasing to $39 \%$ at 5 years. In almost half the cases of HRS, one or more precipitating factors may be identified, including bacterial infections (57\%), gastrointestinal haemorrhage (36\%), and large volume paracentesis (7\%) [7]. Spontaneous bacterial peritonitis (SBP) is the most frequent cause of renal failure in cirrhosis with approximately $30 \%$ of patients with SBP developing ARF [8].

The epidemiology of studies on HRS in the future may display the statistical phenomenon of stage migration, because of alterations to the diagnostic criteria for HRS in 2007. These future trials may demonstrate improved outcomes and mortality figures, a reflection of stage migration, where patients fall into a different diagnostic group associated with a reduced mortality [9].

\section{Pathophysiology and Basic Science}

The pathophysiology of HRS is complex, and yet it seems certain that it is functional in nature and related to three key areas - splanchnic, sympathomemetic and cardiac factors - which will be discussed in more detail.

The evidence to support the functional nature of HRS comes from a number of sources outlined below: (a) initially histological abnormalities are minimal and inconsistent; (b) tubular function and sodium absorption remains intact; (c) kidneys transplanted from patients with HRS can resume normal function in the recipient, and (d) renal function can return in patients with HRS who receive a liver transplant.

HRS and the associated intense renal vasoconstriction is the result of two important physiological changes, which have been shown to occur in advancing liver disease, portal hypertension and acute liver failure. Firstly, there is an increase in the level of endogenous vasodilators and vasoconstrictors. Primarily, vasodilatation affects the splanchnic circulation and vasoconstriction the renal circulation. Secondly, specific cardiac abnormalities develop, leading to a cirrhotic cardiomyopathy, which now appears to be crucial in the development of HRS. This cirrhotic cardiomyopathy is unmasked during stressful events like sepsis compounding the already established dysfunctional circulation, which has evolved as liver disease advances due to the release of the numerous vasoactive mediators.

The Liver and Kidney in Critically Ill Patients
Doppler studies have demonstrated the discrepancy of vasodilatation in the splanchnic circulation and vasoconstriction in muscle, kidney and cerebral circulations [10]. Vasoactive mediators such as nitric oxide (NO), carbon monoxide, endothelin, prostacyclin and glucagon have all been implicated in splanchnic vasodilatation [11, 12]. Evidence to support the role of such mediators has come from studies assessing various antagonists. An endothelin antagonist, in particular, has been shown to improve renal perfusion in patients with HRS, suggesting that it may play a role in renal vasoconstriction. However, due to conflicting results in these mediator antagonist studies, further studies appear necessary to define the individual role they play in HRS [13]. NO has been extensively studied and is believed to play a significant role in the development of renal dysfunction. Animal studies have used NO inhibitors in an induced acute liver failure model in rats and demonstrated a reduction in serum creatinine (SCr) [14].

\section{Splanchnic}

Splanchnic vasodilatation leads to a reduction in the effective circulating blood volume [15], causing a fall in the mean arterial pressure (MAP) and an increase, in an attempt to compensate, in cardiac output. The fall in MAP shifts the renal blood flow (RBF) autoregulation curve to the right rendering it pressure dependant. The normal physiologically range for renal autoregulation is a MAP of $70-75 \mathrm{~mm} \mathrm{Hg}$. The reduction in RBF leads to activation of systemic endogenous vasoconstrictor systems, the sympathetic nervous system, the renin-angiotensin systems and the non-osmotic release of vasopressin. Consequently, there is avid renal sodium retention resulting in ascites, renal water conservation resulting in hyponatraemia and severe renal vasoconstriction leading to HRS.

\section{Sympathomemetic}

The sympathetic nervous system is known to have heightened activity in cirrhotic patients [16]. In a rat model, the liver has been shown to be involved in the regulation of sodium and water excretion by the kidney. The involvement of the liver in the regulation of urine production has been called the 'hepatorenal reflex' [17]. The hepatorenal reflex is thought to be initiated by cirrhosis-induced hepatic portal circulatory changes; both increased portal venous pressure (PVP) and reduced portal venous blood flow (PVBF). It has been difficult to separate these two possible factors as most studies have increased the PVP by occluding the portal vein thus reducing the PVBF.

Blood Purif 2009;28:124-134 
A rat model where a portocaval shunt was formed to perform a controlled reduction in the intrahepatic blood flow was studied and demonstrated a decrease in sodium and water excretion. It was also shown that the administration of an adenosine antagonist reversed the antinatriuretic effect of the reduced PVBF. Adenosine accumulation in the liver increases hepatic artery blood flow and is thought to stimulate a large collection of nerves in the space of Mall. Adenosine may be acting as a neuromediator stimulating these afferent hepatic nerves leading to an increase in sympathetic activation to renal vessels.

It has been suggested that the liver possesses both sensory and homeostatic functions helping to regulate intravascular fluid via the hepatorenal reflex. There are, however, inconsistent findings in human studies regarding the hepatorenal reflex. The hepatorenal reflex does appear to be a pathophysiological response to maintain the effective circulating volume in patients with cirrhosis and reduced portal vein blood flow [18].

\section{Cardiac}

In advanced liver disease, sustained cardiac performance to maintain the hyperdynamic circulation ensuring a continued effective circulating blood volume and RBF appears vital to avoid HRS. However, it is well recognized that there is a greater prevalence of cardiomyopathy in patients with end-stage liver disease than in the general population [19]. Cirrhotic patients, in particular, develop a number of cardiac-specific abnormalities leading to the condition cirrhotic cardiomyopathy. It has been demonstrated that left ventricular work indices such as stroke index, mean systolic ejection rate, left ventricular stroke work are greater than expected in cirrhotic patients at rest, but have attenuated responses to exercise [20]. Histologically, there is myocardial hypertrophy, interstitial and cellular oedema and signs of cellular injury. This leads to left ventricular free wall and, in particular, septal thickening. This is evident to a greater extent in patients with ascites compared to those without [21]. The degree of diastolic dysfunction increases with increasing wall thickness and leads to prolonged isovolumetric relaxation, greater ventricular pressure for the end-diastolic volume. Cardiac failure can then develop acutely with any rapid increases in filling pressure and may explain why patients decompensate after transjugular intrahepatic portosystemic shunts (TIPSS) and transplantation. Other cardiac-specific changes in cirrhotic cardiomyopathy include electrophysiological repolarization changes, enlargement of cardiac chambers, and a reduced response of the heart to direct $\beta$-stimulation ( $\beta$-incompetence).
All these changes occur in the absence of overt congestive failure [22], but render the cirrhotic patient precariously positioned and vulnerable to stressful stimuli like sepsis.

Decompensated liver disease and the development of ascites occur due to the compensatory effects of the activation of vasoconstrictor and antinatriuretic factors attempting to maintain the effective circulating volume. The increasing volume of ascites leads to an increase in intra-abdominal pressure (IAP), which affects the circulation by reducing venous return.

\section{Diagnosis}

The diagnosis of ARF and assessment of glomerular filtration rate (GFR) relies on the measurement of SCr. There remain diagnostic problems with $\mathrm{SCr}$, and these are particularly evident in liver disease. Bilirubin is known to interfere with assays, with hyperbilirubinaemia masking increases in SCr. The degree of error can be up to $57 \%$, but modern autoanalyzers and the use of the end-point Jaffe method have overcome such interference. However, caution should be employed when interpreting $\mathrm{SCr}$ results in the context of hyperbilirubinaemia. The measurement of $\mathrm{SCr}$ is also affected by two particularly important factors, muscle mass and liver synthetic function. The production of creatinine is reduced by $50 \%$ and this in combination with protein malnutrition and muscle wasting result in a significantly lower baseline range for creatinine in advanced liver disease (35-75 $\mu \mathrm{mol} / \mathrm{l})$.

Cirrhotic patients for a given change in GFR tend to have smaller and delayed changes in SCr, underestimating and impairing the recognition of change in GFR [23]. Novel biomarkers for the measurement of GFR are being explored, but a more reliable marker is yet to be found. The best marker of GFR continues to be the SCr, if interpreted with a full understanding of its pitfalls, and it is cheap and widely available.

ARF is a complex disorder, with over 30 definitions, and attempts have been made in recent years to create a uniform standard for diagnosis and classification. Members representing key societies in critical care and nephrology set up the Acute Kidney Injury Network (AKIN) to review the RIFLE criteria used for defining and classifying ARF. Acute kidney injury is the new term used to represent the entire spectrum of ARF, recognizing that an acute decline in kidney function is often secondary to an injury that causes functional or structural changes in the kidneys. 

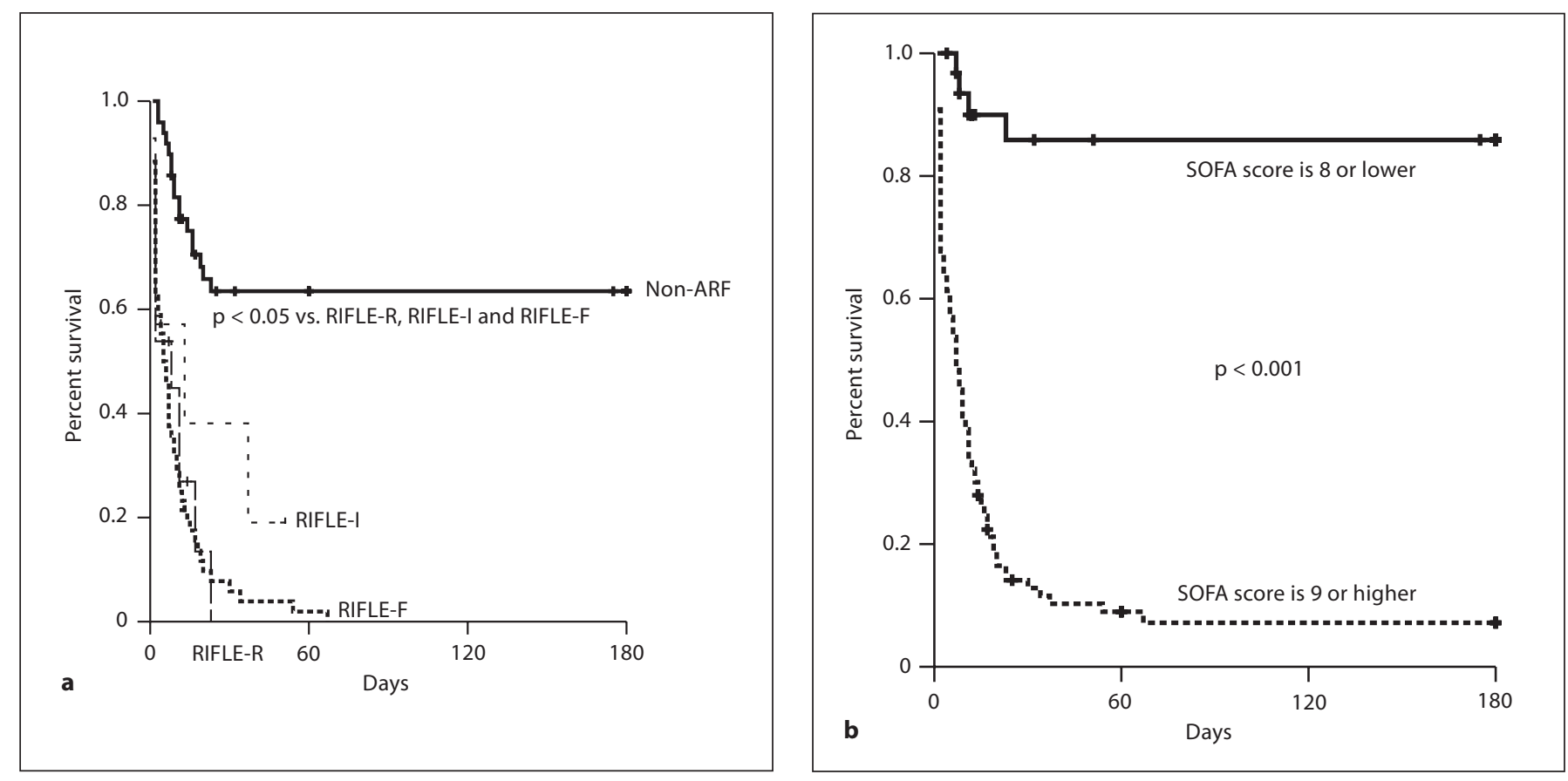

Fig. 1. Cumulative survival rate for 134 critically ill cirrhotic patients based on their RIFLE classification (a) and SOFA (b) on day 1 of ICU admission (permission granted by Y.-C. Chen, MD, Chang Gung Memorial Hospital, Tapei, to use this figure).

The AKIN group decided to revise RIFLE criteria, specifically the 'Risk' category to take into account evidence demonstrating that adverse outcomes are associated with small rises in SCr occurring in $24-48 \mathrm{~h}$, reflecting an acute and significant change in GFR [24]. Patients with a SCr rise $>26.4 \mu \mathrm{mol} / \mathrm{l}$ were included in stage 1 of the AKIN staging system rather than use $>25 \%$ increase from baseline SCr as was the case for RIFLE (table 1). It was assumed this would improve sensitivity and predictability of the definition and classification of AKI. However, a recent study has demonstrated this amounts to only $1 \%$, which is a favourable outcome, because RIFLE has already been validated in over 200,000 patients and is widely used [25]. Therefore, RIFLE and AKI staging are essentially the same with the category 'Risk' being the equivalent to stage 1, 'Injury' stage 2 and 'Failure' stage 3. 'Loss' and 'End-stage' have been removed from AKI, but remain as outcomes. Patients receiving RRT are automatically included in stage 3. Urine output is used as one of the diagnostic criteria, because despite its many shortcomings, it is recognized as a useful measure in critically ill patients as it can often herald the onset of AKI before changes in SCr are identified.

The Liver and Kidney in Critically Ill Patients
Table 1. Classification/staging system for acute kidney injury

\begin{tabular}{lll}
\hline Stage & Increase in serum creatinine & Urine output \\
\hline 1 & $>26.4 \mu \mathrm{mol} / \mathrm{l}$ or $>150-200 \%$ from & $<0.5 \mathrm{ml} / \mathrm{kg} / \mathrm{h}$ \\
& baseline $(1.5$ - to 2 -fold) & $>6 \mathrm{~h}$ \\
\hline 2 & $>200-300 \%$ from baseline & $<0.5 \mathrm{ml} / \mathrm{kg} / \mathrm{h}$ \\
& $(>2$ - to 3 -fold) & $>12 \mathrm{~h}$ \\
\hline 3 & $>300 \%$ from baseline $(>3$-fold $)$ or & $<0.3 \mathrm{ml} / \mathrm{kg} / \mathrm{h}$ \\
& SCr $>354 \mu$ mol/l with acute increase & $>24 \mathrm{~h}$ or \\
& of $44 \mu \mathrm{mol} / \mathrm{l}$ or receiving RRT & anuria for $12 \mathrm{~h}$ \\
\hline
\end{tabular}

The definition and criteria for staging AKI are useful as it alerts us to the significant impact AKI stage has on clinical outcomes (fig. 1). With further validation it could be possible to incorporate one of these AKI staging systems into the criteria for defining HRS, unless a better biomarker of AKI can be found.

Kaplan-Meier curves for survival using RIFLE criteria for AKI in cirrhotic patients who are critically ill have demonstrated this staging system to be a good predictor of hospital survival [26].

Blood Purif 2009;28:124-134 
The diagnosis of HRS is one of exclusion, so investigations should be performed to rule out other common causes of AKI. The characteristic urinary excretory patterns observed in HRS are indistinguishable from prerenal failure or myoglobinuric ATN, where tubular function and the capacity to concentrate remain intact. The measurement of urinary sodium, osmolality or creatinine has little role to play in directing management in clinical practice.

HRS can be diagnosed if various criteria are met in accordance with those agreed by the International Ascites Club. This club has met twice in the last 15 years, and on the most recent occasion in 2006 new criteria were agreed. The changes to the criteria were based on new concepts which had emerged in the intervening years. The key concepts came from Doppler studies evaluating the different changes observed in the various vascular beds and cardiac studies demonstrating cirrhosis-induced cardiomyopathy were also important. There was a recognition that there had been an improvement in survival associated with the use of vasoconstrictor therapy and TIPSS for bacterial infections that caused HRS (table 2).

\section{HRS Classification and Its Problems}

HRS is defined on the basis of a SCr $>130 \mu \mathrm{mol} / 1$ without reference to gender or ethnicity, and at this level a significant number of patients will not fulfil the diagnostic criteria based on creatinine. In advanced liver disease the associated lower baseline $\mathrm{SCr}$ observed results in a large proportion of patients losing $>50 \%$ of function before the diagnosis of HRS could be considered.

HRS is further divided into two subtypes, HRS type 1 and type 2 , which are clinically quite separate and the differences are illustrated in table 3.

\section{Is HRS-1 a Form of AKI?}

HRS-1 is a potentially reversible deterioration of systemic circulatory function or 'vascular failure', involving a combination of factors, but primarily involving splanchnic vasodilatation and renal vasoconstriction, often triggered by a precipitating event. The acute ischaemic injury probably falls somewhere along the spectrum between classical pre-renal failure at one end and the histological definition of ATN at the other. Indeed, a histological study in 5 patients with HRS type 1 assessed kidney biopsy specimens by light and electron microscopy. It demonstrated necrosis of the proximal tubules characterized by swelling, disorganization of the cristae and the ap-

Table 2. Comparison of the criteria for the diagnosis of HRS agreed at the International Ascites Club meetings in 1994 and 2007

$1994 \quad 2007$

Major criteria

Chronic or active liver disease with Cirrhosis with ascites advanced hepatic failure and portal hypertension

Low GFR (SCr $>1.5 \mathrm{mg} / \mathrm{dl} /$

$>130 \mu \mathrm{mol} / \mathrm{l}$ or creatinine clearance

$<40 \mathrm{ml} / \mathrm{min}$ )

\section{Absence of shock, ongoing infection, and current or recent treatment with nephrotoxic drugs. Absence of gastrointestinal fluid losses (repeated vomiting or intense diarrhoea) or renal fluid losses (wt $>500 \mathrm{~g} /$ day in patients with ascites without peripheral oedema or $1,000 \mathrm{~g} /$ day in patients with peripheral oedema}

No sustained improvement in renal function (decrease in SCr to $1.5 \mathrm{mg} / \mathrm{d}$ or less, or increase in creatinine clearance to $40 \mathrm{ml} / \mathrm{min}$ or more) following diuretic withdrawal and expansion of plasma volume with 1.51 of isotonic saline

Proteinuria $<500 \mathrm{mg} /$ day and $\mathrm{no}$ ultrasound evidence of obstructive uropathy or parenchymal disease

\begin{tabular}{ll}
\hline $\begin{array}{l}\text { Minor criteria } \\
\text { Urine volume }<500 \mathrm{ml} / \text { day }\end{array}$ & $\begin{array}{l}\text { No current or recent treatment } \\
\text { with nephrotoxic drugs }\end{array}$ \\
\hline Urine sodium $<10 \mathrm{mmol} / \mathrm{l}$ & \\
\hline Urine osmolality greater than plasma & \\
osmolality & \\
\hline Urine red blood cell $<50 / \mathrm{HPF}$ & \\
\hline Serum sodium concentration & \\
$<130 \mathrm{mmol} / \mathrm{l}$
\end{tabular}

pearance of dark bodies in the mitochondria, coalescence, fragmentation or displacement of the microvilli, loss of plasma membranes, rupture of the basement membranes, and separation of the cells from the basement membranes. Rupture of tubular basement membranes (tubulorrhexis) and mitochondrial dark bodies suggested an ATN due to ischaemia or induced by vasoconstrictor substances [28]. 
Table 3. Comparison of the different clinical types of HRS-1 and -2

\begin{tabular}{lll}
\hline & HRS-1 & HRS-2 \\
\hline Definition & $\begin{array}{l}\text { Doubling of SCr to }>2.5 \mathrm{mg} / \mathrm{dl} \text { or } 225 \mu \mathrm{mol} / \mathrm{l} \\
(50 \% \text { reduction in } \mathrm{CrCl} \text { to }<20 \mathrm{ml} / \mathrm{min})<2 \text { weeks }\end{array}$ & $\begin{array}{l}\text { SCr }>1.25-2.5 \mathrm{mg} / \mathrm{dl} \text { or } 110-225 \mu \mathrm{mol} / \mathrm{l} \\
\text { progressive decline }\end{array}$ \\
\hline Degree of renal impairment & AKI & CKD \\
\hline Clinical pattern & AKI with precipitating event & $\begin{array}{l}\text { Spontaneous onset with associated diuretic } \\
\text { resistant, refractory ascites }\end{array}$ \\
\hline Time course & Acute $<2$ weeks & $4-6$ months \\
\hline Mortality (median survival) & $<1$ month & 6 months \\
\hline
\end{tabular}

Table 4. CKD stages and GFR values (http://www.renal.org/ CKDguide/ckd.html) (see text for details)

\begin{tabular}{ll}
\hline CKD stage & GFR $\left(\mathrm{ml} / \mathrm{min} / 1.73 \mathrm{~m}^{2}\right)$ \\
\hline 1 & $>90$ \\
2 & $60-89$ \\
3 & $30-59$ \\
4 & $15-29$ \\
5 & $<15$ or dialysis \\
\hline
\end{tabular}

The improved understanding of ATN has recognized that there is more than just an upset in haemodynamic autoregulatory mechanisms, which normally preserves the intraglomerular pressure. The contributions of endothelial, leukocyte activation and the release of cytokines causing an oxidative stress at a cellular level are important in the development of kidney injury. The models of AKI in sepsis and HRS-1 seem to be very similar, and the pathological mechanism perhaps explains why improving renal perfusion alone does not always result in improved kidney function. Future therapies aimed at improving $\mathrm{RBF}$, enhancing natriuresis, and dampening down inflammation, free radicals and oxidative stresses are all likely to be important.

HRS-1, unlike HRS-2, is an 'acute' pathology defined as a doubling of $\mathrm{SCr}>225 \mu \mathrm{mol} / \mathrm{l}$ within 2 weeks from the initial defining $\mathrm{SCr}>130 \mu \mathrm{mol} / \mathrm{l}$. This is far removed from the latest acute kidney injury staging system, where stage 1 is defined as a $150-200 \%$ increase from baseline. Stage 1 AKI for patients diagnosed with HRS would equate to a rise in SCr $>195-260 \mu \mathrm{mol} / \mathrm{l}$ within 48 hours. The rate of change between the two classifications varies enormously, 48 hours compared to 2 weeks. Many patients with acute liver disease have a progressive condition evolving over days or weeks exposing them to the increased risk of HRS, which can develop at any point in time during their illness. This, though, should not preclude these patients from being assessed for the insult of acute kidney injury using the AKI staging criteria.

\section{Is HRS-2 a Form of Chronic Kidney Disease (CKD)?}

HRS-2 is perhaps a form of chronic ischaemic nephropathy similar to that encountered in patients with chronic heart failure, which is exacerbated by precipitating events. Patients with liver disease have the additional burden of large volume, often refractory, ascites and a raised IAP. The effect of diuretics and a raised IAP have important effects on kidney haemodynamics, which are significant and may account for the slow deterioration in kidney function.

HRS-2 appears to be a 'chronic' form of kidney disease, which is associated with refractory ascites. Patients with the HRS-2 condition perhaps should be classified using CKD staging criteria, which were developed by the Kidney Disease Outcome Quality Initiative (K/DOQI) workgroup to help manage and stage CKD. It relies on using estimated GFR (eGFR) calculated using the modified diet in renal disease (MDRD) calculation. There are different versions of the MDRD calculated eGFR; the extended version incorporates albumin and urea. The standard MDRD eGFR has a sensitivity of $65-74 \%$ in predicting GFR in liver disease, and is now considered by many to be the 'gold standard' [27].

If HRS-2 is considered a form of CKD, then the scenario of a 45-year-old male with a SCr between 110 and 225 , with an albumin of 16 and an urea of 8 would equate to CKD stage 3-4, if calculated using the MDRD equa- 
tion version 7, for the two extremes of the HRS-2 definition. CKD stages 3-4 are associated with an increased cardiovascular risk and chance of progression to endstage renal failure. The patient in this example with stage 3-4 CKD will have experienced a significant loss in kidney function, and any opportunity to assess the rate of progression and perform a thorough investigation to exclude important underlying parenchymal diseases or exacerbating factors will have been missed (table 4).

\section{Problems with the HRS Criteria}

There are problems with the current definition of HRS and importantly how the subgroups of HRS-1 and HRS-2 are defined and identified. The initial defining criteria of a SCr $>130 \mu \mathrm{mol} / \mathrm{l}$ is not reflective of the lower baseline SCr concentration seen in these patients, and it is not adjusted for gender or ethnicity. HRS should perhaps be divided into acute and chronic HRS. Acute HRS and AKI staging could be employed based on changes in the individual baseline SCr measurement and urine output when available. Chronic HRS could be defined using CKD staging criteria where patients have eGFR calculated using one of the MDRD equations and then management directed in line with current CKD guidelines. The nephrology community has embraced these staging systems for AKI and CKD, acknowledging their pitfalls, but importantly recognizes that they can alert clinicians earlier to patients with renal disease. This is important, because there is an abundance of evidence demonstrating that even a small degree of kidney injury is associated with an increased mortality and that CKD is significantly associated with an increase in cardiovascular disease.

\section{Prevention}

Simple measures may prevent AKI, including the temporary omission of nephrotoxic drugs together with appropriate adjustment of drug doses for the eGFR. Diuretic dosing is especially important in patients with ascites or cardiac failure. Twenty percent of patients with ascites develop moderate renal impairment, related to excessive diuresis and intravascular depletion, which is often rapidly reversible following diuretic withdrawal.

Radiological investigation requiring contrast should be performed once measures to prevent contrast-induced nephropathy have been implemented. There is much debate about the pathogenesis and management of this condition. However, both volume loading with isotonic bicarbonate and the use of the antioxidant agent $\mathrm{N}$-acetyl- cysteine have been shown to reduce the occurrence of contrast-induced nephropathy in patients at risk [29, 30].

The prevention of HRS is limited to targeting the precipitating events and reducing the incidence with which they occur. Orthotopic liver transplantation (OLT) and TIPSS may be useful interventions, but patient selection needs collective careful consideration.

SBP can cause hepatic encephalopathy and HRS-1 and is associated with $30 \%$ hospital mortality despite infection resolution. Volume expansion with albumin $(1.5 \mathrm{~g} / \mathrm{kg}$ day $1,1 \mathrm{~g} / \mathrm{kg}$ day 3 ) has been shown to significantly reduce the incidence of HRS and hospital mortality associated with SBP. The albumin effect is related to improvements in systemic haemodynamics, indicated by plasma renin aldosterone (PRA) suppression. The patients who benefitted in this study were those with a serum bilirubin $>68 \mathrm{mmol} / \mathrm{l}(4 \mathrm{mg} / \mathrm{dl})$ and a SCr $>88.4 \mathrm{mmol} / \mathrm{l}(1 \mathrm{mg} / \mathrm{dl})$ [31]. Trials need to be conducted so that the optimum dosage to be used can be defined more precisely.

Studies into antibiotic prophylaxis for recurrent SBP have demonstrated it to be highly effective and cost-effective at reducing recurrent events. However, the use of antibiotics as primary prophylaxis with norfloxacin is not so clear. A recent study suggested that primary prophylaxis reduces the incidence of SBP, delaying the development of HRS, and improving survival. It would appear though, that further work is still required to establish the clinical and cost effectiveness of primary prophylaxis in this group of patients. This is even more apparent given the associated increased incidence of Gram-positive organisms, which has been shown in those treated with norfloxacin [32].

Many studies have reviewed both the medical and endoscopic therapies that can prevent variceal bleeding. There is no effective therapy for the prevention of varices, but $\beta$-blockade can prevent the enlargement of varices and so reduce the risk of bleeding. They have been shown to clearly reduce the risk of variceal bleeding in moderate to large varices and some evidence, although weak, suggests endoscopic banding is superior to medical therapy [33]. Often the episode of AKI associated with variceal bleeding is related to the period of volume depletion and hypotension.

\section{Management Strategy}

AKI in advanced liver disease whatever the cause should be focused on the early optimization of renal perfusion with the goal of saving nephrons. Strategies be- 
yond resuscitation used for the treatment of HRS have included the use of vasopressors and albumin, TIPSS and OLT.

\section{Haemodynamic Resuscitation}

The Surviving Sepsis Campaign initial resuscitation recommendations, which were developed to help direct therapy in patients with sepsis or sepsis-induced organ dysfunction (shock), provide useful initial targets for patients with AKI, but are not validated for patients with liver disease [34]. There are problems associated with the measurement of central venous pressures and $\mathrm{ScvO}_{2}$ in patients with liver disease. Central venous pressure is elevated by large volume ascites and raised intra-abdominal pressure. $\mathrm{ScvO}_{2}$ is often significant raised in patients with advanced liver disease reflecting the hyperdynamic circulation.

The Surviving Sepsis Campaign recommends commencing resuscitation in any patient who is hypotensive, MAP $<70 \mathrm{~mm} \mathrm{Hg}$, or has an elevated serum lactate $>4 \mathrm{mmol} / \mathrm{l}$, and to give due consideration of management being transferred to a critical care environment. The lactate threshold of $4 \mathrm{mmol} / \mathrm{l}$ in a cirrhotic patient may of course be difficult to interpret, but a rising trend would still suggest significant circulatory disarray.

In patients with advanced liver disease, the issues that cloud the conventional measures of central blood volume and tissue perfusion often necessitate the earlier institution of invasive haemodynamic monitoring. This is especially advisable when there has been failure to achieve a MAP $>65 \mathrm{~mm} \mathrm{Hg}$ or urine output $>0.5 \mathrm{ml} / \mathrm{kg} / \mathrm{min}$ with fluid resuscitation, and the decision to commence inotropic or vasopressor support has been taken. When sepsis is suspected, cultures should be taken from different sites and antibiotics commenced within an hour of diagnosis.

Cirrhotic patients can often present unwell with tense ascites, and this causes intra-abdominal hypertension (IAH). IAH that exceeds $25 \mathrm{~mm} \mathrm{Hg}$ can result in significant compromise to cardiac, respiratory and renal function. Large volume therapeutic paracentesis can help to reduce IAH often immediately improving respiratory mechanics. Support with albumin and vasopressors is essential to prevent exacerbation of the established circulatory disturbances. Small volume paracentesis should sometimes be considered as this may avoid the dramatic effects on the central haemodynamics incurred with higher volume paracentesis.

\section{Orthotopic Liver Transplantation}

The modified end-stage liver disease (MELD) scoring system was developed to predict survival for patients un- dergoing TIPSS. This was then utilized to predict the risk of death within 3 months of diagnosis of advanced liver disease in to order to prioritize these patients for cadaveric liver transplant. In February 2002, the United States adopted the MELD score to rank patients accordingly on transplant waiting lists. The MELD score uses a mathematical formula based on SCr, bilirubin, and INR. MELD scores can range from 6 to 40 (MELD scores $>40$ are all grouped together and receive a score of 40$)$. MELD $=3.8$ [In serum bilirubin $(\mathrm{mg} / \mathrm{dl})]+11.2$ [In INR] + 9.6 [In SCr $(\mathrm{mg} / \mathrm{dl})]+6.4$. MELD has helped those with HRS and a high SCr to gain a higher priority. However, it has been demonstrated that due to the problems with variations in SCr measurement due to high levels of bilirubin, muscle mass, gender and age there are significant variations in MELD scores. Although this may not affect survival prediction score, it can affect ranking on transplant waiting lists [35].

Many patients with HRS-1 are unsuitable for transplantation, because of significant associated organ dysfunction or indeed they die before transplantation is possible. HRS at the time of transplantation is associated with a negative effect on outcome in terms of survival, renal recovery, need for long-term RRT, cost effectiveness and quality of life [36]. Ongoing debate and research is trying to elucidate which of these patients should receive a combined kidney and liver transplant or liver transplant alone.

\section{Vasopressors, Albumin and TIPSS}

Most studies in HRS-1 have been non-randomized and concentrated on the use of vasopressors (midodrine, terlipressin and noradrenaline), octreotide and albumin infusion. Midodrine and noradrenaline are $\alpha$-agonists, which improve systemic blood pressure by increasing systemic vascular resistance. Octreotide antagonizes the splanchnic vasodilators. Terlipressin is a V1 vasopressin agonist, which increases both systemic and splanchnic vascular resistance increasing effective circulating blood volume.

\section{HRS}

The vasopressor studies to date have used mostly bolused terlipressin or continuously infused vasopressin and demonstrated recovery rates for HRS-1 of around $59 \%$. Interestingly, terlipressin in conjunction with albumin infusions have been shown to be superior than terlipressin alone with respect to renal recovery. The combi- 
nation of midodrine and octreotide has also been studied, and resulted in a $49 \%$ recovery rate [37].

One prospective, randomized, double-blind, placebocontrolled clinical trial of terlipressin has been performed. Patients with type 1 HRS were randomized to terlipressin (1 mg every $6 \mathrm{~h}$ ) or placebo, with albumin in both groups. Terlipressin was superior to placebo with reversal rates for HRS-1 of 34 and 13\%, respectively. Transplantation-free survival was similar between study groups, but HRS reversal significantly improved survival at day 180 [38].

The role of TIPSS in HRS-1 has been studied on only a few occasions and restrictive patient selection criteria make it difficult to extrapolate these findings into daily clinical practice. The TIPSS procedure reduces the portal pressure and sympathetic drive, which has been shown to affect renal haemodynamics. Patients with advanced liver disease must be carefully selected for the TIPSS procedure, because the reduction in portal blood flow to the liver can precipitate decompensation of liver function and precipitate encephalopathy.

Wong et al. [39] evaluated the additional improvement of renal function following TIPSS in patients with HRS-1. They selected patients who had improved renal function with medical therapy and enrolled patients for TIPSS therapy, unless they had contraindications for TIPSS insertion. These included an international normalized ratio $>2$, a serum bilirubin $>85 \mu \mathrm{mol} / \mathrm{l}(5 \mathrm{mg} / \mathrm{dl})$, a ChildTurcotte-Pugh score of $\geq 12$, or thrombosed portal vein and active infection within the previous 2 weeks. They showed that patients treated with TIPSS had significant further sustained improvement of renal function and elimination of ascites [40].

\section{HRS-2}

HRS-2 is associated with refractory ascites and progressive renal impairment. Refractory ascites is managed either by paracentesis or TIPSS and only a few trials have been performed comparing these two treatment options. TIPSS is superior to paracentesis in the prevention of refractory ascites. The incidence of gastrointestinal bleeding, infection and AKI are similar for both treatment groups. TIPSS is though associated with an increased incidence of encephalopathy.

The use of vasopressor therapy with albumin and TIPSS alone has been studied for the treatment of HRS-2 in non-randomized trials. Similar improvements for renal function and survival have been found, although the latter would be expected given the natural history of the disease.

\section{Extracorporeal Therapies}

RRT continues to be the mainstay of supportive therapy for patients who, despite aggressive resuscitation, lose the function of all remaining nephrons. Effective RRT corrects metabolic disturbances, fluid overloaded states and problems with thermoregulation.

The molecular adsorbent recirculating system (MARS) is an innovative extracorporeal treatment that is thought to remove albumin-bound vasoactive substances such as NO, tumour necrosis factor, and other proinflammatory cytokines to help transiently recover hepatic function. It has been used in patients with HRS-1 and resulted in clinical improvement in renal function producing a positive effect on 30-day survival in these patients (37.5 vs. $0 \%$ ). This study was small, and they did not receive albumin or terlipressin therapy [41]. Future studies with MARS, vasoactive therapies and albumin will be necessary to evaluate its role further.

\section{Critical Care Issues}

The Liver Intensive Care Unit at King's College Hospital is a specialist 15-bed unit dedicated to the admission and management of patients with liver disease. The patient population consists of both medical and surgical patients with liver disease. The medical patients consist of those who have become critically ill with decompensated cirrhosis or various presentations of acute liver failure. The surgical patients are a mix of those: post-liver transplant, small for size syndromes, liver trauma or following the resection of both primary and secondary hepatic tumours.

\section{Systematic Issues}

\section{Airway}

Encephalopathy in these patients demands protection of the airway. Intubation with an endotracheal tube initially, and at an appropriate and safe time tracheostomy is performed. Rapid sequence intubation is used in patients with ascites, as delayed gastric emptying from increased abdominal pressures increases the risk of aspiration. Tracheostomy allows the reduction of sedation necessary for patients to tolerate the endotracheal tube, which would otherwise delay recovery in these already encephalopathic patients. 


\section{Cardiovascular}

Adequate organ perfusion is maintained by the early use of haemodynamic monitoring to optimize volume resuscitation along with vasopressor use. Steroids are used to help up-regulate adrenoreceptors, and when vasopressor use is significant or prolonged the hypothalamic-pituitary-adrenocortical axis is often assessed using the Synacthen test, despite the known pitfalls. Intra-abdominal pressure (IAP) is monitored using bladder manometers, and paracentesis performed whenever there are resultant circulatory or respiratory issues.

\section{Respiratory}

In critically ill cirrhotic patients there is a wide spectrum of respiratory problems encountered. Ventilator-associated pneumonia occurs despite ventilator care bundles being rigorously implemented. Adult respiratory distress syndrome, hydrothoraces and the hepatopulmonary syndromes are also encountered.

\section{Gastrointestinal}

Gut failure and the associated issues of poor absorption of enteral feeds require early placement of nasojejunal tubes and use of total parenteral nutrition. Supplementation with vitamins and trace element are used be- cause of the increased requirement during critical illness with liver disease and use of continuous renal replacement therapies.

\section{Conclusions}

(1) Renal dysfunction is associated with an increased mortality in patients with cirrhosis. (2) There is a probably higher incidence of CKD - type 2 HRS. (3) Type 1 HRS is similar to that of AKI in sepsis/systemic inflammatory response syndrome with treatment aimed at early central volume expansion. (4) Problems with the definition of HRS make it difficult to use in clinical practice, recent attempts to stage both $\mathrm{AKI}$ and CKD provide potential alternatives to the threshold SCr concentrations currently used. (5) Evidence base for terlipressin and albumin appears to be good. (6) Accurate biomarkers of kidney injury are needed to help diagnose injury to the kidney earlier. (7) TIPSS may be considered in selected patients (CP score $<12$ and early responders to medical therapy). (8) Transplantation is an option, but a high incidence of renal dysfunction in recipients due to immunosuppression and diabetes is often observed.

\section{References}

1 Hoste EAJ, Clermont G, Kersten A, Venkataraman R, Angus DC, De Bacquer D, Kellum JA: RIFLE criteria for acute kidney injury are associated with hospital mortality in critically ill patients: a cohort analysis. Critical Care 2006;10:R73

-2 Ng CKF, Chan MHM, Tai MHL, Lam CWK: Hepatorenal syndrome. Clin Biochem Rev 2007;28:11-17.

-3 Betrosian AP, Douzinas EE, Agarwal B: Acute renal dysfunction in liver diseases. World J Gastroenterol 2007;13:5552-5559.

$\checkmark 4$ Hegarty J, Middleton RJ, Krebs M, et al: Severe acute renal failure in adults: place of care, incidence and outcomes. QJM 2005;98: 661-666.

5 Nash K, Hafeez A, Hou S: Hospital acquired renal insufficiency. Am J Kidney Dis 2002; 39:930-936.

-6 Abosaif NY, Tolba YA, Heap M, Russell J, El Nahas AM: The outcome of acute renal failure in the intensive care unit according to RIFLE: model application, sensitivity, and predictability. Am J Kidney Dis 2005;46: 1038-1048.
7 Gines A, Escorsell A, Gines P, et al: Incidence, predictive factors and prognosis of the hepatorenal syndrome in cirrhosis and ascites. Gastroenterology 1993;105:229_ 236.

8 Gines P, Martin PY, Niederberger M: Prognostic significance of renal dysfunction in cirrhosis. Kidney Int 1997;51(suppl):S77S82.

-9 Angeli P, Merkel C: Pathogenesis and management of hepatorenal syndrome in patients with cirrhosis. J Hepatol 2008;48:93103.

10 Rivolta R, Maggi A, Cazzaniga M, et al: Reduction of renal cortical blood flow assessed by Doppler in cirrhotic patients with refractory ascites. Hepatology 1998;28:12351240.

11 Dagher L, Moore K: The hepatorenal syndrome. Gut 2001;49:729-737.

12 Wiest R, Groszmann RJ: The paradox of nitric oxide in cirrhosis and portal hypertension: too much, not enough. Hepatology 2002;35:478-491.
13 Soper CPR, Latif AB, Bendino MR: Amelioration of hepatorenal syndrome with selective endothelin-A antagonist. Lancet 1996; 347:1842-1843.

14 Islas-Carbajal MC, Covarrubias A, Grijalva G, Alvarez-Rodríguez A, Armendáriz-Borunda J, Rincón-Sánchez AR: Nitric oxide synthases inhibition results in renal failure improvement in cirrhotic rats. Liver Int 2005;25:131-140.

15 Arroyo V, Planas R, Gaya J, et al: Sympathetic nervous activity, renin-angiotensin system and renal excretion of prostaglandin $E_{2}$ in cirrhosis. Relationship to functional renal failure and sodium and water excretion. Eur J Clin Invest 1983;13:271-278.

16 Bichet DG, Van Putten VJ, Schrier RW: Potential role of increased sympathetic activity in impaired sodium and water excretion in cirrhosis. N Engl J Med 1982;307:15521557.

17 Jalan R, Forest EH, Redhead DN, Dillon JF, Hayes PC: Reduction in renal blood flow following acute increase in portal pressure: evidence for the existence of a hepatorenal reflex in man? Gut 1997;40:664-670. 
-18 Ming Z, Smyth DD, Wayne Lautt W: Decreases in portal flow trigger a hepatorenal reflex to inhibit renal sodium and water excretion in rats: role of adenosine. Hepatology 2002;35:167-175.

-19 Nagarakanti R, Whellan D, Rubin S, Mather PJ: Reversible cardiomyopathies. Cardiol Rev 2007;15:178-183.

20 Wong F, Girgrah N, Graba J, Allidina Y, Liu P, Blendis L: The cardiac response to exercise in cirrhosis. Gut 2001;49:268-275.

-21 Valeriano V, Funaro S, Lionetti R, Riggio O, Pulcinelli G, Fiore P, Masini A, De Castro S, Merli M: Modification of cardiac function in cirrhotic patients with and without ascites. Am J Gastroenterol 2000;95:3200-3205.

-22 Liu H, Song D, Lee SS: Cirrhotic cardiomyopathy. Gastroenterol Clin Biol 2002;26: 842-847.

23 Epstein M: Hepatorenal syndrome; in Brady HR, Wilcox CS (eds): Therapy in Nephrology and Hypertension - A Companion to Brenner and Rector's the Kidney, chapt 8. Philadelphia, Saunders, 1999, pp 45-50.

-24 Mehta RL, Kellum JA, Shah SV, Molitori BA, Ronco C, Warnock DG, Levin A, and the Acute Kidney Injury Network (AKJN): Report of an initiative to improve outcomes in acute kidney injury. Critical Care 2007;11: R31.

25 Bagshaw SM, George C, Bellomo R, et al: A comparison of the RIFLE and AKIN criteria for acute kidney injury in critically ill patients. Nephrol Dial Transplant 2008;23: 1203-1210.

-26 Jeng CC, Tsai MH, Tian YC, Lin CY, Yang C, Liu NJ, Lien JM, Chen YC, Fang JT, Chen PC, Yang CW: RIFLE classification can predict short-term prognosis in critically ill cirrhotic patients. Intensive Care Med 2007;33: 1921-1930.
27 Kupin W: Renal Disease Complicating Advanced Liver Disease. Alexandria/VA American Association for the Study of Liver Disease, pp 221-226.

28 Mandal AK, Lansing M, Fahmy A: Acute tubular necrosis in hepatorenal syndrome: an electron microscopy study. Am J Kidney Dis 1982;2:363-374.

-29 Briguori C, Airoldi F, D’Andrea D, Bonizzoni E, Morici N, Focaccio A, Michev I, Montorfano M, Carlino M, Cosgrave J, Ricciardelli B, Colombo A: Renal Insufficiency Following Contrast Media Administration Trial (REMEDIAL): a randomized comparison of three preventive strategies. Circulation 2007;115;1211-1217.

30 Hogan SE, L'Allier P, Chetcuti S, Grossman PM, Nallamothu BK, Duvernoy C, Bates E, Moscucci M, Gurm HS: Current role of sodium bicarbonate-based preprocedural hydration for the prevention of contrast-induced acute kidney injury: a meta-analysis. Am Heart J 2008;156:414-421.

-31 Sort P, Navasa M, Arroyo V, et al: Effect of intravenous albumin on renal impairment and mortality in patients with cirrhosis and spontaneous bacterial peritonitis. N Engl J Med 1999;341:403-409.

32 Fernández J, Navasa M, Planas R, Montoliu S, Monfort D, Soriano G, Vila C, Pardo A, Quintero E, Vargas V, Such J, Ginès P, Arroyo V: Primary prophylaxis of spontaneous bacterial peritonitis delays hepatorenal syndrome and improves survival in cirrhosis. Gastroenterology 2007;133:818-824.

33 Tiani C, Abraldes JG, Bosch J: Portal hypertension: pre-primary and primary prophylaxis of variceal bleeding. Dig Liver Dis 2008; 40:318-327.
34 Dellenger RP, Levy M, Carlet JM, et al: Surviving Sepsis Campaign: international guidelines for management of severe sepsis and septic shock: 2008. Critical Care 2008; 36:296-327.

35 Cholongitas E, Marelli L, Kerry A, Senzolo M, Goodier DW, Nair D, Thomas M, Patch D, Burroughs AK: Different methods of creatinine measurement significantly affect MELD scores. Liver Transpl 2007;13:523529.

36 Pawarode A, Fine DM, Thuluvath PJ: Independent risk factors and natural history of renal dysfunction in liver transplant recipients. Liver Transpl 2003;9:741-747.

37 Solanki P, Chawala A, Garg R, Gupta R, Jain $M$, Sarin SK: Beneficial effects of terlipressin in hepatorenal syndrome: a prospective, randomized placebo-controlled clinical trial. J Gastroenterol Hepatol 2003;18:152-156.

38 Sanyal AJ, Boyer T, Garcia-Tsao G, Regenstein F, Rossaro L, Appenrodt B, Gulberg V, Sigal S, Teuber P; Terlipressin Study Group: A randomized prospective double-blind, placebo-controlled trial of terlipressin for type 1 hepatorenal syndrome. Gastroenterology 2008;134:1360-1368.

>39 Wong F, Pantea L, Sniderman K: Midodrine, octreotide, albumin, and TIPS in selected patients with cirrhosis and type 1 hepatorenal syndrome. Hepatology 2004;40:55-64.

$>40$ Bresing KA, Textor J, Perez J, et al: Longterm outcome after transjugular intrahepatic portosystemic stent-shunt in non-transplant cirrhotics with hepatorenal syndrome: a phase II study. Gut 2000;47:288-295.

41 Mitzner SR, Stange J, Klammt S, et al: Improvement of hepatorenal syndrome with extracorporeal albumin dialysis MARS: results on a prospective, randomized controlled clinical trial. Liver Transpl 2000;6: 277-286. 